\title{
HUMAER: A Test-Bed Environment for Human Factors Investigations in the Aeronautic Domain
}

\author{
D. H. Arjoni ${ }^{1}$, W. R. de Oliveira $^{2}$, L. G. Trabasso ${ }^{3}$ and E. Villani ${ }^{4} *$ \\ *Division of Mechanical Engineering, Aeronautics Institute of Technology, São José dos Campos, SP/Brazil \\ E-mail: arjoni@ita.br' ${ }^{1}$ wesleyro@ita.br², gonzaga@ita.br ${ }^{3}$, evillani@ita.br ${ }^{4}$
}

\begin{abstract}
This work presents the Human Factors in Aeronautics laboratory (HUMAER), from the Aeronautics Institute of Technology (ITA). This laboratory is a test-bed environment built around the SIVOR - the flight simulator with a robotic motion platform from ITA - with the purpose of evaluating the different relations among the pilot cognitive, psychological and physiological states and the aircraft's human-computer interfaces and cybernetics. The lab is comprised of different virtual reality technology and non-intrusive physiologic sensor technology that can be installed on the pilot to monitor its psycho-cognitive state from a range of objective measures. Considering some characteristics of SIVOR's cockpit, like the motioncueing ability and the haptics stimuli generated from the force-feedback inceptors and shakers, some of the main prospective research lines to be explored by the ITA's team are presented according to the key technologies made available at HUMAER. To exemplify some of the current research made within the lab, some findings on pilot perception and workload evaluation from previous referenced investigations are reported.
\end{abstract}

Keywords: Human factors, aeronautic training, flight simulation, physiologic sensors.

\section{Introduction}

Pilot training concerned with upset-recovery, pilot-induced oscillations (PIO), pre-stall conditions identification and new single-pilot cockpit paradigms are all some of the quite recent interests from the main aerospace community that have prompted academics worldwide towards human factors research [1-3]. Broadly speaking, human factors refer to a classical interdisciplinary research area concerned with the interaction of humans with other technologies, environments and systems [4-5]. In the aerospace context, this is translated into the evaluation of the diverse relations among the pilot cognitive, psychological and physiological states and the aircraft's human-computer interfaces and cybernetics [6].

With the aim of evaluating and developing solutions in this research field, there is HUMAER, the Human Factors in Aeronautics laboratory from the Aeronautics Institute of Technology (ITA), located in Brazil. This laboratory is a test-bed environment built around the SIVOR, a full-motion flight simulation system with a robotic motion platform from ITA (Figure 1). Besides the heavy-payload robotic arm and the embedded visual system, SIVOR presents a reasonable trade-off between the realism of the intern cockpit interface and its customization (Figure 2) [7]. The SIVOR cockpit is also enhanced by means of some haptics stimuli that can be generated from the force-feedback inceptors and unit shakers.

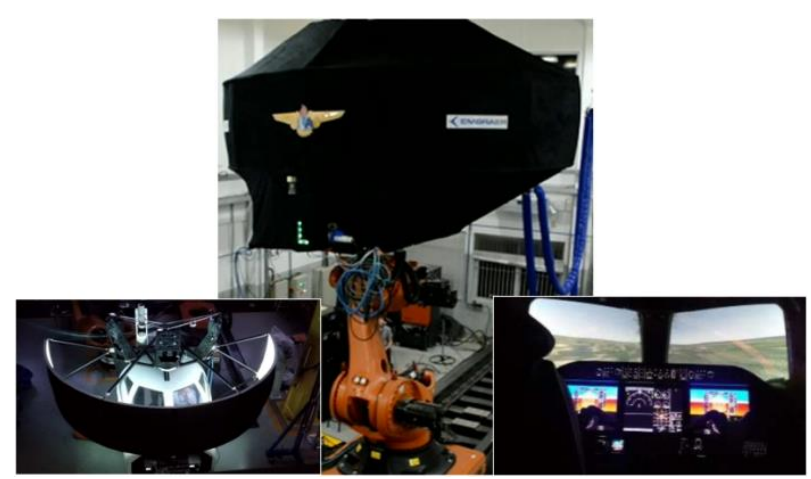

Figure 1: SIVOR - robotic flight simulator.

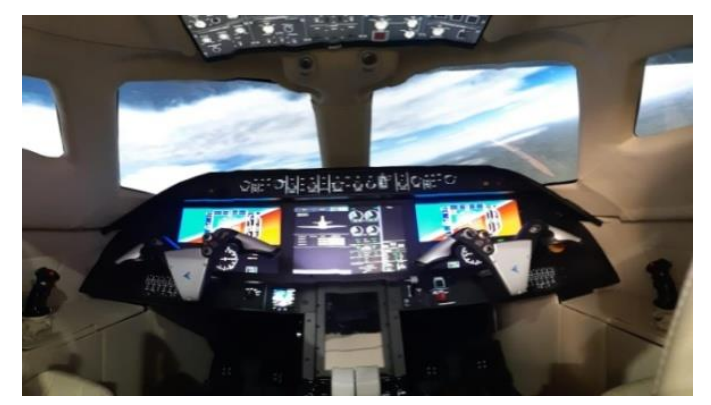

Figure 2: SIVOR's flexible cockpit with main flight deck, yoke and stick inceptors and virtual overhead panel. 
HUMAER is based on the concept of monitoring the pilot's psycho-cognitive state from a range of objective measures gathered from non-intrusive physiologic sensors that can be installed on the subject. Some of the main sensor technologies that enable current research at HUMAER are presented in this work, providing a further overview on the research topics developed in the laboratory. Besides that, some selected results from previous investigations are discussed. To accomplish this goal, this work is organized as follows: section 2 presents the laboratory's infrastructure; section 3 details some of the main prospective research topics of interest of ITA's team; section 4 provides some measurement results from previous investigations; and section 5 brings up the main discussions and conclusions.

\section{HUMAER's equipment and systems}

HUMAER's main sensory equipment is built around sensors, modules and subsystems from Tobii ${ }^{\mathrm{TM}}$, TEA $^{\mathrm{TM}}$ and Wearable Sensing ${ }^{\mathrm{TM}}$. All of them are detailed in Table 1, Table 2 and Table 3, respectively.

\begin{tabular}{c|c|c}
\multicolumn{2}{c}{ Table 1-Tobii ${ }^{T M}$ pro equipment. } \\
\hline Equipment & Image & Description \\
\hline $\begin{array}{c}\text { Tobiipro X3- } \\
120^{\circledR}\end{array}$ & & Eye-tracking bar \\
\hline $\begin{array}{c}\text { Tobiipro } \\
\text { Glasses 2 } \\
\text { Premium } \\
\text { Package }\end{array}$ & & $\begin{array}{c}\text { Package: eye-tracking } \\
\text { glasses with 50 Hz- } \\
\text { record unit, calibration } \\
\text { software, Prolab license }\end{array}$ \\
\hline $\begin{array}{c}\text { Tobii pro VR } \\
\text { Integration } \\
\text { with HTC } \\
\text { Vive }\end{array}$ & & $\begin{array}{c}\text { 120 Hz eye-tracking } \\
\text { glasses with HTC Vive } \\
\text { integration }\end{array}$ \\
\hline
\end{tabular}

Table 2 - TEA ${ }^{T M}$ S.A.R.L. equipment, including CaptivePhysio and CaptivMotion.

\begin{tabular}{|c|c|c|}
\hline Equipment & Image & Description \\
\hline CAPTIV GSR & & Galvanic Skin Response \\
\hline $\begin{array}{c}\text { CAPTIV } \\
\text { Temperature }\end{array}$ & & Body-temperature sensor \\
\hline $\begin{array}{l}\text { CAPTIV TCE } \\
\text { Respiration }\end{array}$ & & $\begin{array}{l}\text { Thoracic contraction- } \\
\text { expansion sensor for } \\
\text { breathing monitoring }\end{array}$ \\
\hline CAPTIV ECG & & $\begin{array}{l}\text { Electrocardiograph } \\
\text { sensor }\end{array}$ \\
\hline
\end{tabular}

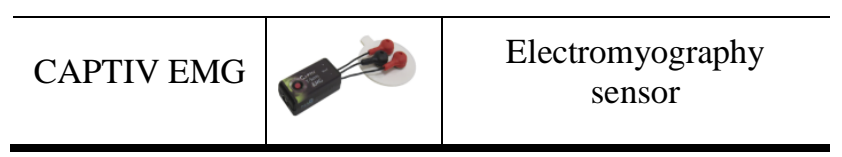

Table 3 - Wearable Sensing $L L C^{T M}$ equipment.

\begin{tabular}{c|c|c}
\hline Equipment & Image & Description \\
\hline $\begin{array}{c}\text { DSI-24 System } \\
\text { EEG }\end{array}$ & $\begin{array}{c}\text { Electroencephalography } \\
\text { dry headband sensor } \\
\text { with } 24 \text { channels. }\end{array}$ & \\
\hline
\end{tabular}

Some general use for all of these sensors are [8-10]: ECG (electrocardiographic sensor) for heart-rate monitoring; ECG (electroencephalographic sensor) for brain activity monitoring; GSR (galvanic ski-response sensor) for sweating monitoring according to stress and awareness state; EMG (electromyography sensor) for muscular activity monitoring; TCE (thoracic contraction-expansion sensor) for respiration monitoring; eye-tracker glasses/bar for blinking, focused vision area and pupil dilation measurements.

They can be all synchronized together using the Captiv ${ }^{\circledR}$ application to help in the identification of the pilot workload and awareness in front of the flexible SIVOR's cockpit, which can also be adapted by means of some augmented reality technology detailed in Table 4 (Besides the Tobii HTC Vive in Table 1, which is also a virtual reality glasses).

Table 4-Augmented and Mixed reality equipments.

\begin{tabular}{c|c|c}
\hline Equipment & Image & Description \\
\hline $\begin{array}{c}\text { Moverio BT- } \\
350^{\circledR}\end{array}$ & & $\begin{array}{c}\text { Epson }{ }^{\circledR} \text { smart- } \\
\text { glasses. }\end{array}$ \\
\hline Meta 2 & & $\begin{array}{c}{ }^{\circledR} \\
\text { Meta }{ }^{\circledR} \text { Augmented } \\
\text { Reality Glasses with } \\
\text { development kit. } \\
\text { Unity license. }\end{array}$ \\
\hline HoloLens ${ }^{\circledR}$ & & $\begin{array}{c}\text { Microsoft } \\
\text { HoloLens }\end{array}$ \\
& & $\begin{array}{c}\text { Hor for } \\
\text { holographic mixed } \\
\text { reality }\end{array}$ \\
\hline
\end{tabular}

The SIVOR's cabin can be further instrumented with an Inertial Measurement Unit (IMU) and unit shakers. In the following subsection, some research topics current under interest of the development team are detailed in regard to the technology made available at HUMAER.

\section{HUMAER's research topics}

Five research fronts are identified by the ITA's development team to use the aforementioned HUMAER's equipments within the SIVOR's investigations demands: 
1) Evaluation of the effectiveness of the robotic motion platform with different strategies

Since some of the main approaches to evaluate the fidelity of motion-cueing in a flight simulator are based on qualitative pilot's feedback [11], the emphasis in this area is to use objective cognitive measures for this kind of investigation. Some topics are:

a) Comparative analysis of the washout filter algorithms used to control the robotic platform using additional excitation form the SIVOR's shaker units and measuring the platform's accelerations using the SIVOR's IMU (Inertial Measurement Unit) and the pilot's level of perception using the GSR, ECG and eye-tracking's pupil dilation measures.

b) Assessment of the correlation between the visual system projection and the platform's motion using the pilot eyetracking and the IMU.

2) Evaluation of the cockpit's Human-Machine Interfaces (HMI) relations to the pilot workload and situation awareness

The idea of this research front is to evaluate pilot awareness upon different workload failure situations. Measurements of the pilot's response time, fatigue and flight performance are intended to be done using the HUMAER's sensory equipment. Some topics are:

a) Assessment of the current EMB505 (Legacy 500) symbology to failure, communication and reaction using parallel measurements from EEG, GSR, ECG and TCE. The eye-tracking technology can be used to evaluate the pilot's vision area, gaze blink, head-up and head-down times.

b) Evaluation of the more important contextual information in the Principal Function Displays (PFDs) and Multifunctional Displays (MFDs) during some failures using the eye-tracking bar, ECG, GSR and TCE.

c) Flight quality assessment in regard to the different profiles of inceptors' force feedback stimuli using EMG, GSR, TCE, ECG and eye-tracking system for pupil dilation measurements.

d) Pilot's fatigue and workload delimitation and quantification using the original cockpit's HMI's resources upon failure conditions. Equipments to be used: ECG, GSR, EEG, TCE, Temperature sensors and eye-tracking glasses.

e) Pilot's fatigue and workload delimitation and quantification using the additional mixed reality stimuli using further symbology from the HoloLens and Meta 2 glasses upon failure conditions. Equipments to be used: ECG, GSR, EEG, TCE, Temperature sensors, HoloLens and Meta 2.

f) Cockpit's preliminary design review using 3D virtual reality environments. Equipments: ECG, TCE and eyetracking with Tobii Vive HTC glasses.

3) Identification of Pilot Induced Oscillations (PIO) events trough pilot cognitive feedback

Broadly speaking, this research area concerns with the identification of the pilot-induced oscillations mechanisms and its respective detection by the pilot using the available cockpit's HMIs and contextual information feedback [12]. The main idea is to perform flight campaigns in SIVOR with pilots that present different experience inducing some characteristic inceptors' disturbances and trimming conditions that drive pilots to PIO. The following topics can be further investigated along the test:

a) Pilot behavior and physiologic state within the phases prior and after PIO events.

b) Pilot behavior and physiologic state during the attitude recovery and PIO mitigation.

c) Techniques for pilot training on PIO avoidance.

d) Development of flight control laws to detect and mitigate PIO events.

All of these research topics are supposed to use parallel measurements of: GSR; ECG; TCE; EMG; Temperature; EEG; and eye-tracker glasses (to monitor the focused area of the pilots vision, response times, gaze blink, head-up and head-down times).

\section{4) Upset conditions identification and upset-recovery training.}

An aircraft upset is defined when, during the flight, it is unintentionally driven to exceed the parameters (attitude or airspeed) normally experienced in line operations or training. It represents a dangerous condition since it may result in Loss of Controllability (LOC) of the aircraft according to some system failure, adverse environment or excessive inadvertent attitude command [13]. In the context of the SIVOR flight simulator, there exists some demands in evaluating possible training conditions where the pilot can identify an upset and recovery from that. The following topics can be further investigated within HUMAER test-bed:

a) Development of additional control laws and contextual HMI feedback to communicate upset conditions to the pilot.

b) Delimitation and quantification of pilot stress and fatigue in conditions of upset-recovery.

c) Analysis of correlation between qualitative psychobehavioral feedback and physiologic measurements of pilots during trimmed upset-recovery maneuver.

d) Training on behavioral recovery techniques in emergency upset situations due to systems failures.

All of these research topics are supposed to use parallel measurements of: GSR; ECG; TCE; EMG; Temperature; EEG; and eye-tracker glasses (to monitor the focused area of the pilots vision, response times, gaze blink, head-up and head-down times).

\section{5) Design of new cockpit concepts and HMI}

a) Application of virtual and augmented reality tools for enhancing pilot's situation awareness through novel HMI and contextual symbology stimuli, interactive cockpit, single pilot cockpit. This topic is supposed to use: HoloLens Mixed Reality Glasses; Meta 2 Augmented Reality glasses; Moverio BT-350 Smart Glasses; VR Vive Tobii Pro; Unity 
Engine and $\mathrm{CAD}$ tools for 3D modeling; eye-tracking glasses; EEG, ECG; GSR; TCE and EMG.

\section{HUMAER's research samples}

Let us present some samples of previous results [11, 14-17] from researches developed along the setup and development of the SIVOR flight simulator and HUMAER test-bed. In addition, to evince the potential of the sensory equipment, we provide some measurements from some sensors gathered along a regular flight training session in SIVOR.

\subsection{Model-based evaluations}

One of the major concerns of the SIVOR's development team during its implementation phase was to evaluate the effectiveness of the motion-cue strategy with a robotic arm and a linear rail unit for the fight training fidelity. Prior the setups of the HUMAER testbed, the analyses were eminently based on the qualitative pilots' assertions [11, 17] or model-based evaluations that further considered the human's physiologic perception system models [14-16].
In [14-16] full model-based approaches aimed at objectively evaluating the pilot perception on the robot motion are presented. All of them found on validated aircraft and robot dynamic models but lacked robustness on the final conclusions because of the use of a model of the pilot. Notwithstanding, some important results were presented, and one of them is depicted in Figure 3 [14].

Figure 3 depicts what is further understood as the pilot perception on the accelerations impelled by the simulator along a running take-off followed by its abortion.

\subsection{Advantages of SIVOR for human factors research}

According to the results presented in the previous subsection, since the SIVOR differs in its setup from "ordinary" full flight simulators (FFS) which usually use a Gough-Stewart platform [19], it has been of great importance to stablish an understanding of the influence of the robot arm and rail unit dynamics on the simulation quality. Basically, recent results developed by the SIVOR's team has evinced some advantages of this kind of approach for high-gain maneuvers.

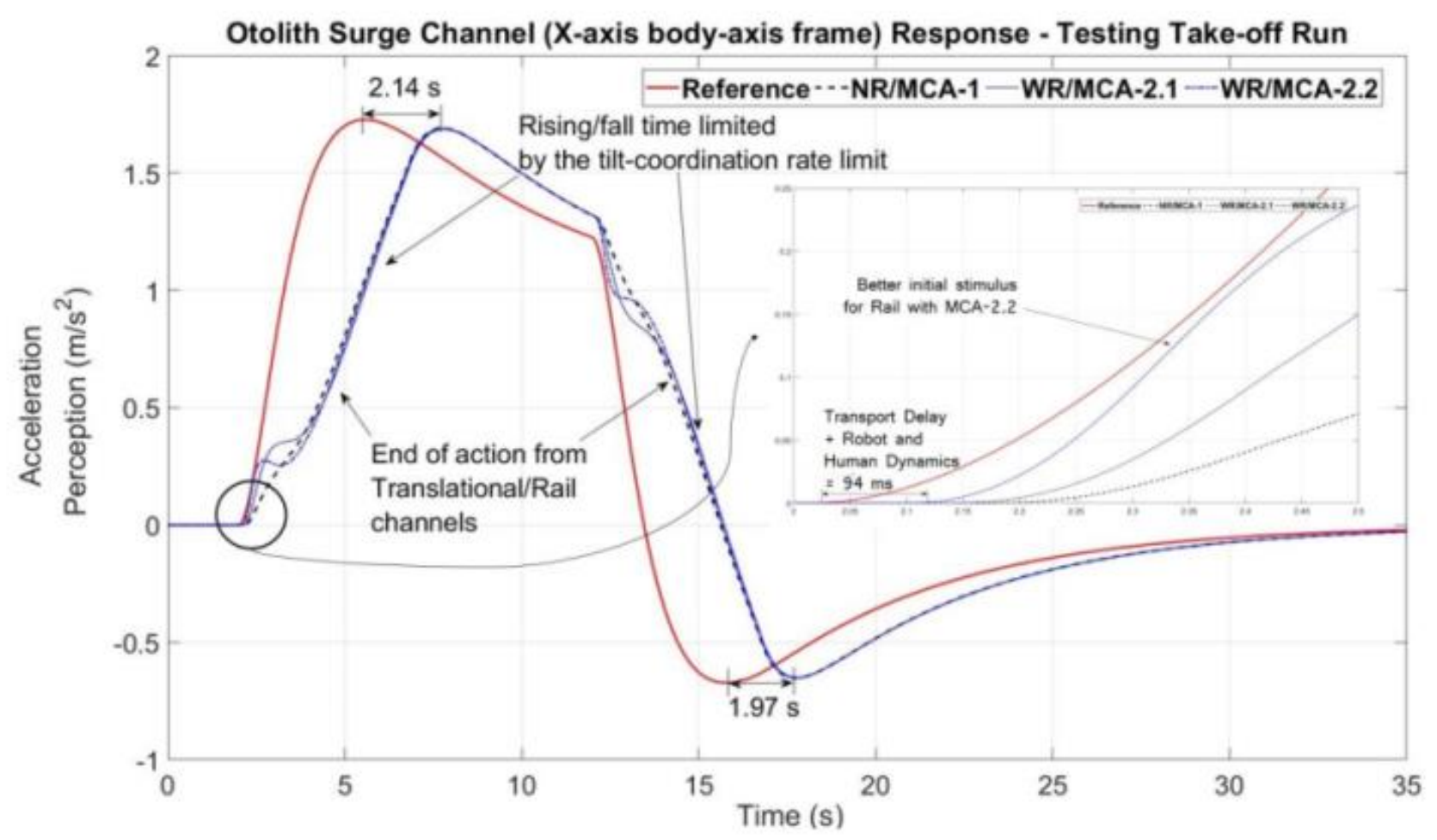

Figure 3: model-based comparison of pilot perceived surge acceleration under different simulator configurations [14].

These stand for those maneuvers where the aircraft is subject to more constant or continued accelerations, like in a reject take-off or a descent pitch-down, which may also precede a LOC event. This directly meets the objectives of the research lines on human factors presented in this paper. Quite newer results in the real SIVOR platform with qualitative pilot evaluations indicate that the rail unit being used as compared in [14], to excite low-frequency surge accelerations (WR/MCA-2.2 in Figure 3), has provided better stimulus. Besides of that, more objective measures have been obtained in the real platform with an IMU (inertial measurement unit) from the HUMAER's infrastructure. They are depicted in Figure 4 for an aircraft Dutch-roll transient due to a rudder command performed within the simulator with the WR/MCA-2.2 active. This result evinces, at least from a first qualitative inspection, a good agreement between the surge acceleration generated by the aircraft and that gathered within the simulator from the IMU. The difference in scales are related to the scale factor used in the initial tests with the washout filter. 


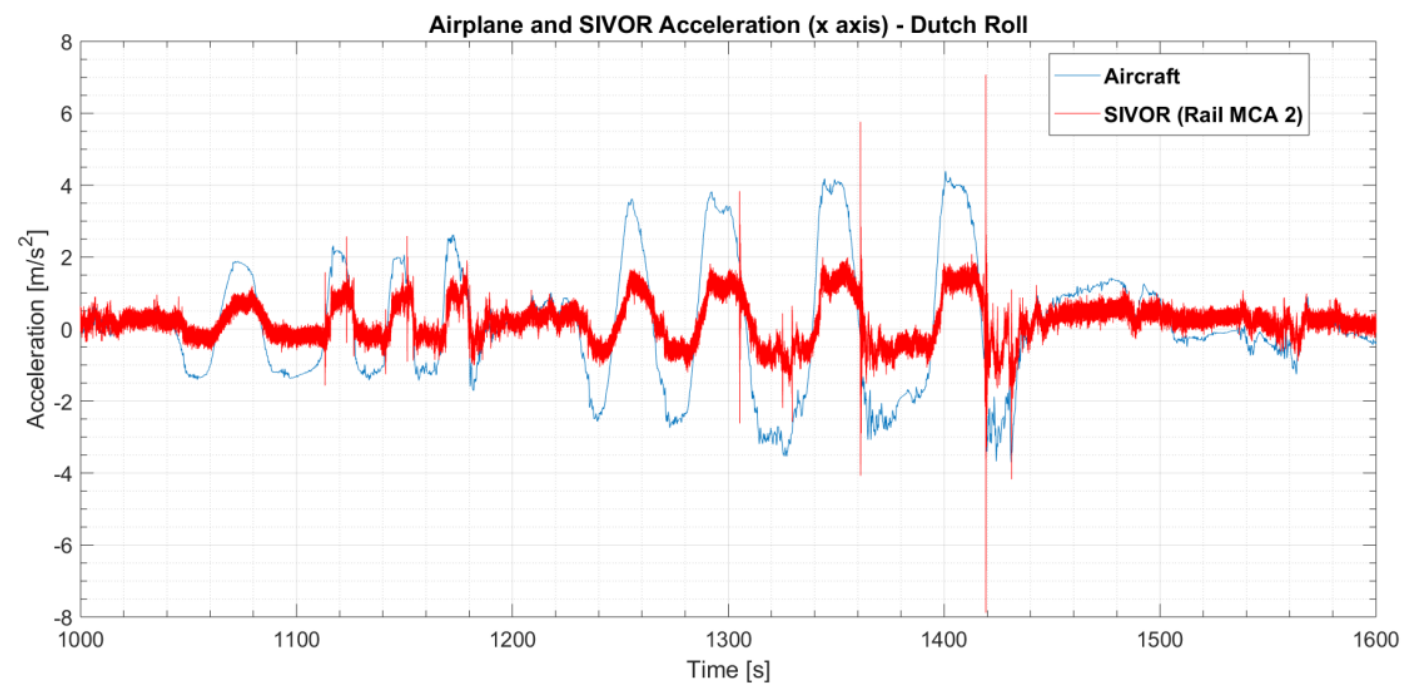

Figure 4: Comparison of the accelerations from the aircraft (blue) and the SIVOR (red). The latter is measured with an IMU.

\subsection{Workload investigations}

Some previous works from the SIVOR team aimed to use human factors related variables to access the effectiveness of the motion of the flight simulator [17]. In these works, performance and workload were estimated through a collection of aircraft dataset including joystick movements, altitude, attitude, airspeed, among others. The results pointed to a necessity of analysing these variables, considering a deeper psychological and biological approach.

To increase the practical understanding towards the workload and performance, the team designed an experiment to access how the pilot interact with the aircraft when exposed to different levels of workload [18]. The results detected changes of performance on variables regarded to the piloting command among workload levels, such as rate of climb, especially when the aircraft was experiencing an abnormal situation. With the setup of the HUMAER test-bed, some more objective measurements with real pilots have been collected, which are presented in the following subsection.

\subsection{Samples of physiologic measurements}

Some physiologic measurements using the ECG, GSR and eye-tracking glasses have been taken in a subject piloting the simulated EMB505 (Legacy $^{\circledR}$ 500) with a simple regular session training comprised of (using the stick inceptor): take-off run, altitude stabilization and pitch and roll maneuvers. The pilot was asked to perform a coordinated bank at some point while keeping control of altitude and airspeed. Figure 5 presents the heat-map distribution of the focused vision area of the pilot during this occurrence. The redder is a given area the more the pilot focused in it.

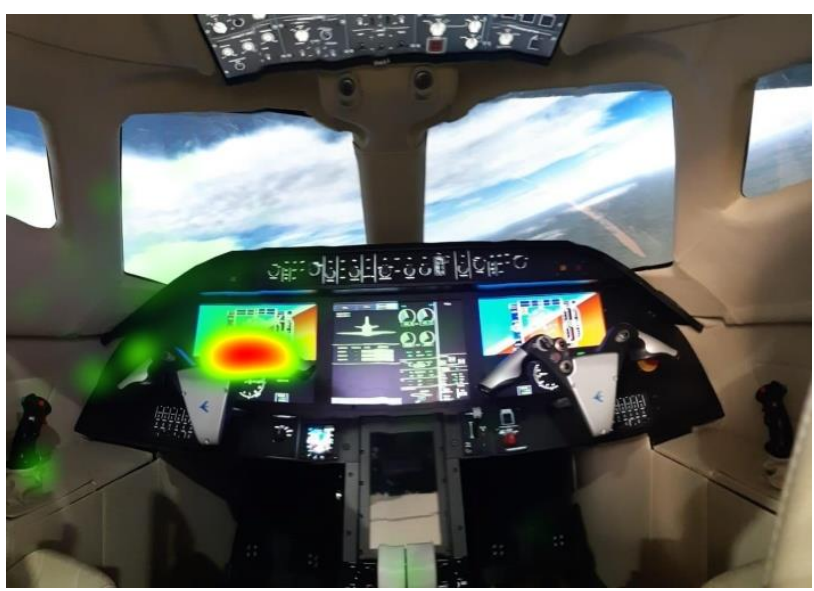

Figure 5: heat map of the pilot's vision focused area during an attempt of compensating a system failure.

As it should be expected, the incipient result present in Figure 5 evinced the pilot focused its vision more in the feedback of the PFD and in position he was impelling to the stick grip, what shows his attempts for keep track of the established limits.

Additional data, such as event occurrence, and a simplified psychological state estimation, can be extracted from the ECG and GSR sensors curves (Figure 6), which were synchronized to the whole system and the eye-tracking glasses.

Although rather initial, these sample results evince the potential of the HUMAER's infrastructure to evaluate different abnormal conditions during regular flight training. All the investigations that are mentioned in this paper could be further developed considering more objective physiologic measures. 


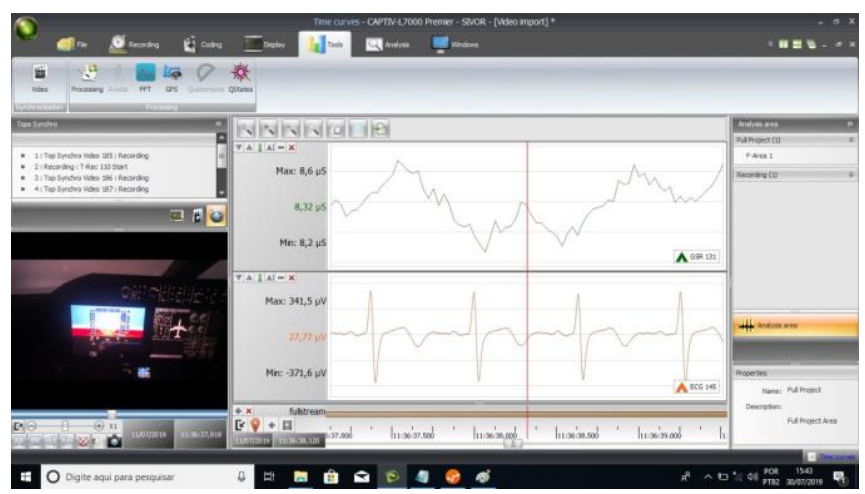

Figure 6: GSR (upper) and ECG (lower) signals acquired in the Captiv ${ }^{\circledR}$ software.

\section{Conclusions}

The main purpose of this work was to introduce the ITA's HUMAER lab, as a potential test-bed for Human Factors investigation in aeronautics. Besides of having been built around the flexible SIVOR flight simulation environment, the laboratory is equipped with relevant and modern physiologic sensor and virtual/mixed/augmented reality technology. Some research topics that has been emphasized by the ITA's team includes upset-recovery training, design of new cockpit concepts and HMI, identification of Pilot Induced Oscillations (PIO) events, evaluation of the motioncueing strategies for flight simulation and of the cockpit's HMIs. Some results from previous works have been presented as well as some measurements gathered with some of the exampled sensors. This paper is expected to bring incitements to the aerospace community in Human Factors investigations.

\section{References}

[1] D. Acosta, Y. Yildiz, R. Craun, S. Beard, M. Leonard, G. Hardy and M. Weinstein, "Piloted Evaluation of a Control Allocation Technique to Recover from PilotInduced Oscillations," JOURNAL OF AIRCRAFT, vol. 52, pp. 130-140, 2015.

[2] J. Bürki-Cohen, "Technical Challenges of Upset Recovery Training: Simulating the Element of Surprise," em AIAA Modeling and Simulation Technologies Conference, Ontario, 2010.

[3] J. Liu, A. Gardi, S. Ramasamy, Y. Lim and R. Sabatini, "Cognitive pilot-aircraft interface for single-pilot operations," Knowledge-Based Systems, 1092016.

[4] G. Salvendy, Handbook of Human Factors and Ergonomics, New Jersey: Wiley, 2012.

[5] F. H. Hawkins, Human Factors in Flight, New York: Routledge, 2016.

[6] J. Wise, D. Hopkin e D. Garland, Handbook of Aviation Human Factors, Taylor \& Francis Group, 2009.

[7] Da silva, E. T. et. al. Flight simulator assisted by a robotic motion plataform. In: Proceedings of AIAA SciTech Forum, 7-11 January, 2019, San-Diego/USA. AIAA: 2019. DOI: 10.2514/6.2019-0435.
[8] TEAErgo, “TEA Ergo," [Online]. Available: http://teaergo.com/wp/?lang=en.

[9] “DSI 24," Wearable Sensing, [Online]. Available: https://wearablesensing.com/products/dsi-24/.

[10] Tobii, “Tobii," [Online]. Available: https://www.tobii.com/.

[11] Natal, G. S. et. al. Implementation Analysis of a Washout Filter on a Robotic Flight Simulator - a Case Study. J. Aerosp. Technol. Manag. [online]. 2019, vol.11, e0919. Epub 14-Jan-2019. ISSN 2175-9146. http://dx.doi.org/10.5028/jatm.v11.978.

[12] R. A. Hess, "Unified theory for aircraft handling qualities and adverse aircraft-pilot coupling," Journal of Guidance, Control, and Dynamics, vol. 20, n⿳⺈, 6 . 1141-1148, 1997.

[13] J. E. Wilborn and V. J. Foster, "Defining Commercial Transport Loss-of-Control: A," in AIAA Atmospheric Flight Mechanics Conference and Exhibit, Rhode Island, 2004. J. E. Wilborn and V. J. Foster, "Defining Commercial Transport Loss-of-Control: A," in AIAA Atmospheric Flight Mechanics Conference and Exhibit, Rhode Island, 2004.

[14] Oliveira et. al. Evaluation of the pilot perception in a robotic flight simulator with and without a linear unit. In: Proceedings of AIAA SciTech Forum, 7-11 January, 2019, San-Diego/USA. AIAA: 2019. DOI: 10.2514/6.2019-0713.

[15] Alves Jr. et. al. An Integrated Mode Based Development to Robotic Motion Flight Simulator. In: Proceedings of AIAA SciTech Forum, 7-11 January, 2019, San-Diego/USA. AIAA: 2019. DOI: $10.2514 / 6.2019-0435$.

[16] Hidalgo, D.; Rodamillans, G. and Oliveira, W. R. Characteristic helicopter flight mechanics and motion cueing integration into the sivor robotic flight simulator: an objective approach. In: Proceedings of 31st Congress of ICAS, 09-14 Septembr, 2018, Beleo Horizonte/Brazil. ICAS: 2018.

[17] D. H. Arjoni, A. Kraemer e E. Villani, "Evolution of an Experiment to Assess Pilot Behaviour in Flight," em International Congress of Mechanical Engineering, Curitiba, 2017.

[18] D. H. Arjoni, E. Villani, M. Rodríguez, A. Matheus, A. Alemida , G. Rocha, L. Gonzaga and D. Hidalgo, "Experimental Analysis of Flight Performance Under Workload Variations," in 31st Congress of the International Council of the Aeronautical Sciences, Belo Horizonte, 2018.

[19] Becerra-Vargas, M. and Belo, E. M., "Robust Control of Flight Simulator Motion Base," Journal of Guidance, Control, and Dynamics, Vol. 34, No. 5, 2011, pp. 1519 1528.doi: doi.org/10.2514/1.53301. 